\title{
Effects of two exercise protocols on postural balance of elderly women: a randomized controlled trial
}

\author{
Laiana Sepúlveda de Andrade Mesquita ${ }^{1,3,5^{*}}$, Fabiana Texeira de Carvalho ${ }^{1,3}$, Lara Sepúlveda de Andrade Freire ${ }^{4}$ \\ Osmar Pinto Neto ${ }^{1,2}$ and Renato Amaro Zângaro ${ }^{1,2}$
}

\begin{abstract}
Background: The aging process reduces both sensory capabilities and the capabilities of the motor systems responsible for postural control, resulting in a high number of falls among the elderly. Some therapeutic interventions can directly interrupt this process, including physical exercise. This study compares and examines the effects of two exercise protocols on the balance of elderly women.
\end{abstract}

Methods: Elderly women who participated in a local church project $(n=63)$ were randomly divided into three groups: the proprioceptive neuromuscular facilitation group (PNFG), Pilates group (PG), and control group (CG). Of the 63 women, 58 completed the program. A training program involving 50-min sessions was performed in the PNFG and PG three times a week for 4 weeks. The elderly women in the CG received no intervention and continued with their daily activities. Stabilometric parameters, the Berg Balance Scale score, functional reach test, and timed up and go test (TUG test) were assessed before and 1 month after participation.

Results: In the comparison among groups, the women in the PNFG showed a significant reduction in most of the stabilometric parameters evaluated and better Berg Balance Scale score, functional reach test result, and TUG test result than did women in the CG $(p<0.05)$. Women in the PG showed significantly better performance on the functional reach test and TUG test than did women in the CG $(p<0.05)$.

Conclusions: Women in the PNFG showed significantly better static and dynamic balance than did women in the CG. Women in the PG also showed better dynamic balance than did women in the CG. However, no significant differences were observed in any of the balance variables assessed between the PNFG and PG.

Trial registration: clinicaltrials.gov, number NCT02278731

Keywords: Movement techniques, Exercise therapy, Elderly, Physical activity, Rehabilitation

\section{Background}

Maintenance of the body's balance is attributed to the postural control system, which involves motor, sensory and nervous system functions [1]. As age progresses, the degree of body sway increases even in simple postural forms, such as an upright posture, causing elderly individuals to sway more than young adults [2].

Changes associated with the aging process, especially those associated with balance, start around the age of

\footnotetext{
* Correspondence: laianasepulveda@hotmail.com

'Universidade Camilo Castelo Branco -Unicastelo, São Paulo, SP, Brazil

${ }^{3}$ Universidade Estadual do Piauí- UESPI, Teresina, PI, Brazil

Full list of author information is available at the end of the article
}

45 years and affect the sensory system (visual, vestibular, and somatosensory) and physical attributes (flexibility, strength, balance, and coordination) [3]. These changes also affect reaction time, causing the elderly to develop many balance-related issues. An improvement in balance control with regular exercise is a good strategy with which to successfully reduce the incidence of falls in this population $[4,5]$.

Several studies have verified the effect of proprioceptive exercise in increasing postural balance by increasing or decreasing body sway [4, 6]. Among several exercise protocols demonstrated to reduce the risk of falls in this population, proprioceptive neuromuscular facilitation

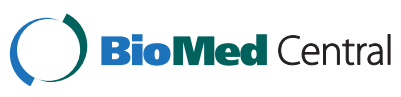

(c) 2015 Mesquita et al.; licensee BioMed Central. This is an Open Access article distributed under the terms of the Creative Commons Attribution License (http://creativecommons.org/licenses/by/4.0), which permits unrestricted use, distribution, and reproduction in any medium, provided the original work is properly credited. The Creative Commons Public Domain Dedication waiver (http://creativecommons.org/publicdomain/zero/1.0/) applies to the data made available in this article, unless otherwise stated. 
(PNF) is particularly compelling because exercise programs aiming to improve balance in the elderly must involve coordination and proprioception activities in addition to strengthening $[4,7]$. Some studies have indicated that PNF exercises are able to improve muscle strength and balance in the elderly [7, 8]. This approach to therapeutic exercises uses specific diagonal movement patterns, reproducing functional movements such as gait, to improve muscle strength and flexibility. It also uses sensory cues such as tactile, visual, and auditory stimuli to improve control and neuromuscular function $[9,10]$.

In addition to PNF exercises, the Pilates method, developed by Joseph Pilates, combines strength and flexibility training and has become increasingly popular, especially in rehabilitation programs [11]. One of the main objectives in Pilates is to strengthen the core muscles, also known as the "powerhouse". This triggers stabilization of the back and pelvic muscles, maintains adequate spinal alignment against gravity, and provides support for limb movements [11]. Several studies have shown that when applied to the elderly, Pilates improves strength, mobility, coordination, and balance [12-14]. Alternatively, some contradictory results have been demonstrated. For example, Bird et al. [15] did not demonstrate significant differences in balance between a Pilates group and control group.

Considering the aforementioned information, we conclude that both PNF exercises and Pilates can improve the complex interaction among the systems responsible for postural balance in different ways. PNF exercises are performed with direct help from a physiotherapist who offers several stimuli to compensate for sensorial deficiencies in the elderly. In the Pilates method, however, the individual must continently control their movements [11] with indirect help from the physiotherapist, who only provides guidance on how to perform the movement.

Thus, Pilates and PNF are interesting choices for the elderly. Although performed differently, both methods stimulate the proprioceptive system, are easily reproduced and performed, and are low in cost. Few randomized controlled studies have investigated the influence of these types of exercises on the balance of the elderly population, and no studies to date have compared the efficacy of Pilates and PNF in improving static and dynamic balance in aged women.

Consequently, the aim of this study was to conduct a randomized controlled trial to investigate and comparethe effect of both exercise methods on the static and dynamic postural balance variables in elderly women, thus identifying alternatives to prevent falls and promoting functional independency.

\section{Methods}

\section{Study participants}

This study involved a convenience sample of older women belonging to a church project named "Pão dos
Pobres". The church was located near the physiotherapy department of a public hospital in Teresina, where the assessments and exercise sessions were conducted. Women who were sedentary as evaluated using the International Physical Activity Questionnaire and aged 60 to 80 years were included in the sample.

The exclusion criteria were any orthopedic, cardiovascular, vestibular, psychological, neurological, or other impairment that would not allow for the execution of all study tasks. A geriatrician who volunteered for the project examined all of the women before the evaluation and intervention.

The study was approved by the local ethics committee (Instituto Educacional Santo Agostinho, approval number 644.367), and all women provided written informed consent to participate. The study is registered on clinicaltrials.gov (number NCT02278731).

\section{Study design}

In total, 150 women involved in the church project were invited to participate in the trial. However, only 90 were interested and therefore evaluated. After application of the inclusion and exclusion criteria, 63 women who could perform all proposed tasks were enrolled.

Each participant was randomly assigned by an independent researcher to either the Pilates group (PG), which involved 1 month of training in the Pilates method three times a week; the PNF group (PNFG), which involved 1 month of training in the PNF method three times a week; or the control group (CG), which involved no intervention (the participants continued their daily activities for 1 month). Three patients from the CG were unable to complete all evaluation tasks and one from the PG and one from the PNFG had more than two absences from the sessions and were thus excluded from the study analysis. The final sample distribution included 20 elderly women in the PNFG, 20 in the PG, and 18 in the CG. Fig. 1 shows the protocol distribution.

The calculations to establish the sample size were done using the software G*Power 3.1.9.2 with one-way ANOVA test in three groups. The significance level was set at $\alpha=0.05$ and power at $80 \%$. Partial eta squared $\left(\eta_{\mathrm{p}} 2=0.153\right)$ was used to measure the effect size, estimated at 0.425 , according to the numbers set for the Timed Up and Go Test in a previous study [16]. The power analysis showed a minimum of 57 individuals (total sample size) were necessary to verify a significant intervention effect and 58 women completed the trial.

The delivery sessions lasted an average of $50 \mathrm{~min}$. The Pilates and PNF protocols were delivered with progressive levels of difficulty to minimize the risk of pain or falls. The trial was performed from May to June 2014. Evaluations were performed $24 \mathrm{~h}$ before beginning the intervention and $24 \mathrm{~h}$ after its end. The protocols were 


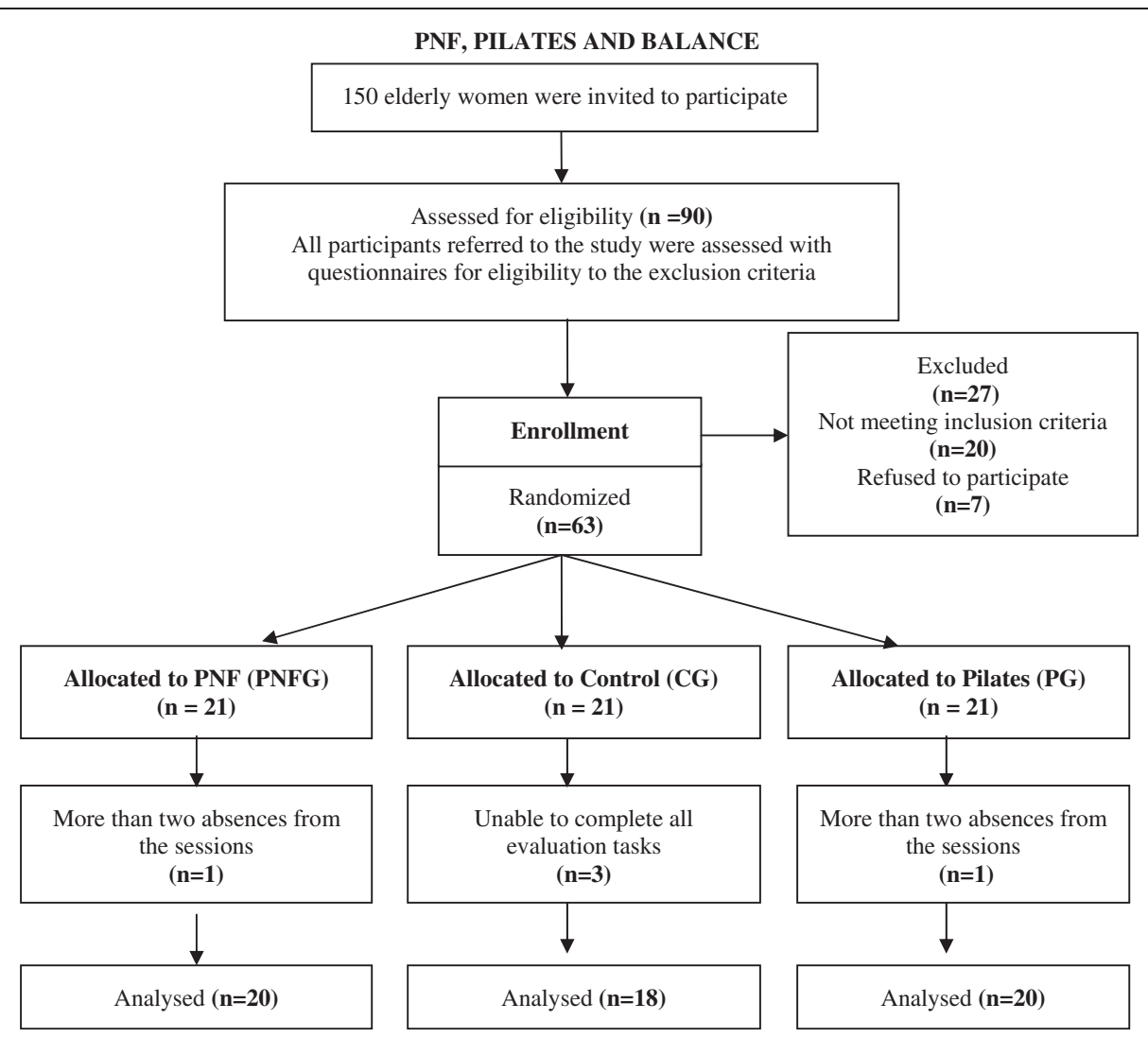

Fig. 1 Study diagram and flow of participants

delivered at the same previously determined time at a room temperature of $25^{\circ} \mathrm{C}$.

\section{Outcome measures}

Data collection and data entry were conducted by research assistants who were blinded to the treatment conditions. Exercises were performed by two physiotherapy professionals. The PNFG provider was certified in PNF, and the PG provider was certified in Pilates.

\section{Stabilometry}

Data were collected using an electronic baropodometer (S-PLATE) comprising a force platform with 1600 sensors, active surface of $400 \times 400 \mathrm{~mm}$ measuring $610 \times$ $580 \times 4 \mathrm{~mm}$ and $6.8 \mathrm{~kg}$ at the University Exercise Clinic in Teresina-PI. The women were oriented in the standing position on the platform; they were barefoot and stood with bipedal support and feet hip-width apart. Furthermore, they were instructed to keep their eyes open while staring at a specific point marked on the wall at eye level, arms relaxed on their sides, for $30 \mathrm{~s}$. Women using corrective lenses wore them during the test. The platform was demarcated on the first attempt using adhesive tape, on which the women positioned their heels to assure the same basis throughout the tests, as suggested by Teixeira et al. [17]. The center-of-pressure (COP) sway kinetic variables analyzed with reference to balance were the total displacement oscillation (TDO), amplitude of displacement of the center of pressure in the anterior-posterior plane (ACPap), amplitude of displacement of the center of pressure in the mid-lateral plane (ACPml), displacement area (AREA), anterior-posterior average speed (AAS), mid-lateral average speed (MAS), and total average speed (TAS).

\section{TUG test}

The TUG test was performed to assess functional mobility regarding speed, agility, and dynamic balance of the elderly women according to Podsiadlo and Richardson [18]. This test assesses the level of mobility of the individual by measuring the time in seconds required to stand up from a chair (seat height of $45 \mathrm{~cm}$ ) without the help of the arms, walk a distance of $3 \mathrm{~m}$, turn around, and return to the chair. At the beginning of the test, the participants' backs must touch the chair, and at the end of the test, the participants return to this position. Time was measured from the "go" command to return to the initial position. The test was performed once for familiarization prior to the timed trial. The women were 
instructed to perform the test as fast as possible while maintaining a comfortable speed to avoid accidents.

\section{Functional reach test}

The functional reach test was performed to analyze dynamic balance. The women were barefoot and instructed to position themselves orthostatically with their lateral body perpendicular to the wall but not touching the wall, feet parallel in a comfortable position, shoulders flexed at $90^{\circ}$ (close to the wall), elbows extended, and hands closed. A measuring tape was fixed horizontally on the wall, parallel to the floor, and positioned at the height of the participant's acromion. The initial measurement was taken at the position at which the third metacarpal met the measuring tape. The women were instructed to lean forward as much as possible without losing balance or taking a step, and the distance was measured as the displacement of the tape from the initial measurement at the third metacarpal to the final measure [19].

\section{Berg balance test}

The Berg Balance Test utilizes a scale of 14 common tasks involving static and dynamic balance, such as reaching, spinning, standing on one foot, and getting up. Each task is given 4 points on an ordinal scale, ranging from 0 (unable to perform the task) to 4 (performs the task independently). The scores of the 14 tasks are summed to yield a total score ranging from 0 to 56 points. Higher scores indicate better performance, while scores of $\leq 45$ are predictive of falls [20].

\section{PNF intervention}

The PNF diagonal patterns of movement were selected considering all the basic facilitation procedures, including resistance, manual pressure, traction, stretch and approximation reflexes, and visual and verbal stimulation. The patterns were consistent with the three specific principles of PNF: rhythmic initiation, sustentation and relaxation, and reversal of antagonists. Initially, the PNF hold-relax stretching technique was performed on the upper and lower limbs. The exercises were then started on the upper limbs in a bilaterally symmetrical agonist pattern: external flexion-abduction-rotation and internal extension-adduction-rotation; external flexion-adductionrotation and internal extension-abduction-rotation. The procedure was then immediately performed on the lower limbs in a bilaterally symmetrical antagonist pattern: external flexion-adduction-rotation and internal extensionabduction-rotation (diagonal gait); internal flexion-abductionrotation and internal extension-adduction-rotation, both diagonals involving variant flexion and extension of the knee [9]. With the woman in a lateral decubitus position, scapular and pelvic girdle exercises were performed in a combined symmetrical and reciprocal manner in the anterior-elevation-posterior-depression diagonal [9]. The researcher offered gradually increasing resistance throughout the range of motion, and the women thus gained strength. Delivery sessions were individualized. During the first week, 1 set of 10 repetitions for each diagonal was performed; in the second week, 2 sets of 10 repetitions were performed; and in the third and fourth weeks, 3 sets of 10 repetitions were performed.

\section{Pilates intervention}

The Pilates protocol involved the performance of Pilates exercises on the ground. Stretches were performed on the upper body and lower limbs before the exercises. Exercises that involved the range of motion and strength of the upper limbs, trunk, and lower limbs were subsequently performed. All exercises were associated with breathing and contraction of the transverse abdominal muscles and were performed in different positions. The number of repetitions and level resistance were increased throughout the study using a Swiss ball, theraband, and magic circle. The exercise sessions were delivered in groups with up to three participants (Table 1).

\section{Data analysis}

A per protocol analysis was used to determine the most efficient of two exercise protocols (Pilates and PNF), lasting four weeks each, in a prearranged population of elderly women. Thus, the women with more than two absences from the sessions were excluded from the analysis. Data were processed and analyzed using PASW Statistics for Windows, Version 18.0. (SPSS Inc., Chicago, IL). Means and standard deviations are presented. First, the Shapiro-Wilk test was used to evaluate the normality of the variables and determine which tests would be used. For the variables following a normal distribution, the paired $t$ test was used to compare two means (withingroup comparisons), and repeated-measures ANOVA was used to compare three means (between-group comparisons). The Wilcoxon test was used on some stabilometric variables that did not follow the normal distribution. The differences in functional test scores and stabilometric variables were calculated for each woman by subtracting the pretraining data from the post-training data in the PG, PNFG, and CG. Repeated-measures ANOVA followed by post-hoc Tukey's test was used to determine differences in the means of each variable between the groups. A p-value of $<0.05$ was considered statistically significant.

\section{Results}

Sixty three elderly women were eligible for inclusion and 58 participants completed the program: 20 in the PNFG (age $=68.5 \pm 5.4$ years), 20 in the PG (age $=67.3 \pm$ 4.9 years) and 18 in the CG (age $=71.5 \pm 6.2$ years). No 
Table 1 Pilates intervention

\begin{tabular}{|c|c|}
\hline FIRST WEEK & REPETITIONS \\
\hline Elevate the ball using upper limbs in dorsal position & 5 \\
\hline Move the ball laterally to the right and left with the heels & 5 \\
\hline Leap over the ball while seated & 5 \\
\hline Belly dance & 5 \\
\hline Bent-knee fall-out & 5 \\
\hline Circle using legs in dorsal position & 5 \\
\hline Bridge & 5 \\
\hline Flex elbow against the wall & 5 \\
\hline \multicolumn{2}{|l|}{ SECOND WEEK } \\
\hline Elevate the ball using upper limbs in dorsal position & 10 \\
\hline Move the ball laterally to the right and left with the heels & 10 \\
\hline Leap over the ball while seated and lifting a foot & 10 \\
\hline Walk on the ball while seated & 10 \\
\hline Four-footed & 10 \\
\hline Circle with legs in dorsal position & 10 \\
\hline Bridge & 10 \\
\hline Flex elbow against the wall while lifting a foot & 5 \\
\hline \multicolumn{2}{|l|}{ THIRD WEEK } \\
\hline Front/Back & 5 \\
\hline Up/Down & 5 \\
\hline Bicycle & 8 \\
\hline Single leg circles, lying down with use of theraband & 8 \\
\hline $\begin{array}{l}\text { Sit on the ball, place theraband under feet, and perform } \\
\text { shoulder flexion }\end{array}$ & 10 \\
\hline Practice magic circle & 10 \\
\hline Elevator & 10 \\
\hline Lift the ball in the air & 10 \\
\hline Four-footed on the ball while lifting hand/foot, alternating sides & 10 \\
\hline Bridge while lifting a foot & 10 \\
\hline Flex elbow against the wall while lifting a foot & 10 \\
\hline Extend knee with magic circle & 10 \\
\hline \multicolumn{2}{|l|}{ FOURTH WEEK } \\
\hline Front/Back & 10 \\
\hline Up/Down & 10 \\
\hline Bicycle & 10 \\
\hline Single leg circles, lying down with use of theraband & 10 \\
\hline $\begin{array}{l}\text { Sit on the ball, place theraband under feet, and perform } \\
\text { shoulder flexion }\end{array}$ & 10 \\
\hline Practice magic circle & 10 \\
\hline Elevator & 10 \\
\hline Lift the ball in the air & 10 \\
\hline Four-footed on the ball, lifting hand/foot and alternating sides & 10 \\
\hline Bridge while lifting a foot & 10 \\
\hline Flex elbow against the wall while lifting a foot & 10 \\
\hline Extend knee with magic circle & 10 \\
\hline
\end{tabular}

differences in age, weight, height and body mass index were found between the three groups (Table 2).

A total of 12 sessions were scheduled for each participant from the Pilates and PNF groups. And all the women included in the analysis attended all the sessions.

\section{Stabilometric data}

The between-group comparisons showed that the PNF group had greater reductions in four of the seven sway measures than the control group (Table 3). No significant differences were found between the Pilates and control groups in any of the sway measures. In the within-group comparisons, there were no significant differences in the COP variables between the Pilates and control groups. However, the PNF group had greater reductions in five of the seven sway measures than the control group (Table 4).

\section{Functional tests}

In the between-group comparisons, women in the PNFG and PG exhibited improved performance in the TUG test and functional reach test compared with women in the CG. The Berg Balance Scale scores improved in the PNFG when compared with the CG $(p=0.005)$. No significant differences in any functional test results were observed between the PG and PNFG $(p>0.05)$ (Table 3).

In a within-group comparison, women in both the PNFG and PG showed significant improvements in the functional reach test, TUG test, and Berg Balance Scale scores. Women in the CG did not show significant differences in the evaluated parameters (Table 5).

\section{Discussion}

This study represents the first controlled evaluation comparing two physiotherapy treatments in the balance of elderly women: Pilates and PNF. The results suggest that in 12 sessions, both methods improved parameters related to the postural balance of elderly women; however, there were no differences between the PNFG and PG. There was a greater reduction in COP sway, verified through stabilometric parameters, in the PNFG than in the CG. The PNFG also showed significant improvements in all functional tests performed. Although women in the PG did not show a significant reduction in COPsway, they

Table 2 Homogeneity of women among the groups $(n=58)$

\begin{tabular}{lllll}
\hline & $\begin{array}{l}\text { Control } \\
(\mathrm{n}=18)\end{array}$ & $\begin{array}{l}\text { PNF } \\
(\mathrm{n}=20)\end{array}$ & $\begin{array}{l}\text { Pilates } \\
(\mathrm{n}=20)\end{array}$ & $\mathrm{p}^{*}$ \\
\hline Age (years) & $71.5 \pm 6.2$ & $68.5 \pm 5.4$ & $67.3 \pm 4.9$ & 0.065 \\
Weight $(\mathrm{kg})$ & $55.7 \pm 5.7$ & $58.4 \pm 9.3$ & $61.0 \pm 9.3$ & 0.164 \\
Height $(\mathrm{cm})$ & $148.3 \pm 7.0$ & $152.7 \pm 5.5$ & $153.2 \pm 5.9$ & 0.335 \\
Body mass index $\left(\mathrm{kg} / \mathrm{m}^{2}\right)$ & $25.4 \pm 2.9$ & $25.1 \pm 5.6$ & $26.0 \pm 3.5$ & 0.747 \\
\hline
\end{tabular}

Data are presented as mean \pm standard deviation ${ }^{*}$ ANOVA 
Table 3 Comparative data of stabilometric and functional capacity (between-group comparison)

\begin{tabular}{lllll}
\hline & $\begin{array}{l}\text { Control } \\
(\text { Pos-Pre })\end{array}$ & $\begin{array}{l}\text { PNF } \\
\text { (Pos-Pre) }\end{array}$ & $\begin{array}{l}\text { Pilates } \\
\text { (Pos-Pre) }\end{array}$ & $p$ \\
\hline TODO $(\mathrm{mm})$ & $148.8 \pm 396.3$ & $-165.9 \pm 259.2^{*}$ & $-34.7 \pm 230.9$ & 0.008 \\
ACPap $(\mathrm{mm})$ & $2.6 \pm 5.5$ & $-0.9 \pm 5.2$ & $0.4 \pm 4.0$ & 0.099 \\
ACPml $(\mathrm{mm})$ & $0.6 \pm 6.0$ & $-4.2 \pm 7.2$ & $-2.4 \pm 5.4$ & 0.069 \\
AREA $\left(\mathrm{mm}^{2}\right)$ & $40.0 \pm 101.0$ & $-37.2 \pm 51.6^{*}$ & $-11.7 \pm 38.6$ & 0.003 \\
AAS $(\mathrm{mm} / \mathrm{s})$ & $4.4 \pm 10.2$ & $-1.6 \pm 9.1$ & $0.6 \pm 6.7$ & 0.115 \\
MAS $(\mathrm{mm} / \mathrm{s})$ & $1.7 \pm 9.2$ & $-6.1 \pm 5.6^{*}$ & $-2.1 \pm 4.9$ & 0.003 \\
TAS $(\mathrm{mm} / \mathrm{s})$ & $5.0 \pm 13.2$ & $-5.5 \pm 8.6^{*}$ & $-1.2 \pm 7.7$ & 0.008 \\
BERG & $0.5 \pm 2.7$ & $4.1 \pm 4.5^{*}$ & $2.0 \pm 1.9$ & 0.005 \\
TAF $(\mathrm{cm})$ & $0.3 \pm 7.9$ & $9.2 \pm 4.8^{*}$ & $8.8 \pm 5.0^{*}$ & $<0.001$ \\
TUG $(\mathrm{sec})$ & $0.1 \pm 3.7$ & $-2.6 \pm 1.3^{*}$ & $-3.6 \pm 2.3^{*}$ & $<0.001$ \\
\hline
\end{tabular}

Data arepresented as mean \pm standard deviation of the pre- to post-test differences calculated as post minus pre so that positive means indicate an increase over time *Significant difference compared with control group by Tukey's post-hoc test after ANOVA, $p<0.05$

TDO: total displacement oscillation, ACPap: amplitude of displacement of the center of pressure in the anterior-posterior plane, ACPml: amplitude of

displacement of the center of pressure in the mid-lateral plane, AREA: displacement area, AAS: anterior-posterior average speed, MAS: mid-lateral average speed, TAS: total average speed

BERG: Berg Balance Scale score, TAF: functional reach test, TUG: timed up and go test

showed a significant improvement in the functional reach test and TUG test performance.

Several stabilometric parameters derived from the COP have been used to quantify changes in balance. The selection of these parameters has been controversial in the literature; there are conflicting opinions about which parameters are more sensitive to fluctuations in the COP. Understanding these variables is essential for analysis of balance [21].

Fujimoto et al. [22] found that the area of oscillation may indirectly reflect the function of the central and peripheral vestibular systems due to reduced visual and somatosensory input. In another study, the authors examined the reliability of COP measures, and the average speed was the most frequently used and most reliable parameter with which to evaluate the body sway [23]. This parameter was also more sensitive in discriminating the effects of manipulative treatments and challenges of balancing tasks for both young adults and healthy elderly participants [24]. The amplitude of COP displacement in the anterior-posterior and mid-lateral planes was also frequently used to verify the balance performance after proprioceptive training [15, 25]. Ruhe et al. [23] noted that although the average speed has good reliability across different studies, no single measure of COP appeared to be significantly more reliable than the others.

Although no significant differences were shown between the PNFG and PG, use of the PNF protocol in elderly women proved to have positive effects; several
Table 4 Stabilometric results of elderly women before and after (within-group comparison) Pilates and PNF training or usual activity

\begin{tabular}{llll}
\hline & Pre & Post & P \\
\hline Control & & & \\
TDO & $701.5 \pm 259.8$ & $853.9 \pm 432.7$ & $0.129^{\mathrm{t}}$ \\
ACPap & $11.1 \pm 4.4$ & $13.7 \pm 6.6$ & $0.063^{\mathrm{t}}$ \\
ACPml & $8.6 \pm 3.8$ & $9.2 \pm 4.8$ & $0.660^{\mathrm{t}}$ \\
AREA & $62.6 \pm 36.3$ & $102.6 \pm 100.6$ & $0.660^{\mathrm{t}}$ \\
AAS & $17.2 \pm 7.0$ & $21.5 \pm 12.1$ & $0.086^{\mathrm{t}}$ \\
MAS & $12.9 \pm 5.7$ & $14.6 \pm 8.0$ & $0.446^{\mathrm{t}}$ \\
TAS & $23.5 \pm 8.7$ & $28.5 \pm 14.4$ & $0.129^{\mathrm{t}}$ \\
PNF & & & \\
TDO & $820.6 \pm 367.0$ & $654.6 \pm 350.6$ & $0.019^{\mathrm{w}}$ \\
ACPap & $11.7 \pm 4.7$ & $10.7 \pm 4.1$ & $0.601^{\mathrm{t}}$ \\
ACPml & $11.5 \pm 8.2$ & $7.3 \pm 5.4$ & $0.001^{\mathrm{w}}$ \\
AREA & $97.5 \pm 57.5$ & $60.3 \pm 98.3$ & $0.003^{\mathrm{w}}$ \\
AAS & $18.8 \pm 8.6$ & $17.2 \pm 7.6$ & $0.654^{\mathrm{w}}$ \\
MAS & $16.2 \pm 9.2$ & $10.1 \pm 8.0$ & $<0.001^{\mathrm{w}}$ \\
TAS & $27.4 \pm 12.2$ & $16.4 \pm 11.7$ & $0.019^{\mathrm{w}}$ \\
Pilates & & & \\
TODO & $629.5 \pm 213.6$ & $594.6 \pm 235.4$ & $0.510^{\mathrm{t}}$ \\
ACPap & $8.9 \pm 3.3$ & $9.3 \pm 2.5$ & $0.601^{\mathrm{w}}$ \\
ACPml & $9.4 \pm 5.4$ & $7.0 \pm 2.7$ & $0.117^{\mathrm{w}}$ \\
AREA & $55.8 \pm 37.6$ & $44.1 \pm 32.1$ & $0.191^{\mathrm{t}}$ \\
AAS & $14.4 \pm 4.6$ & $15.0 \pm 6.5$ & $0.701^{\mathrm{t}}$ \\
MAS & $12.3 \pm 6.2$ & $10.2 \pm 4.8$ & $0.072^{\mathrm{t}}$ \\
TAS & $21.0 \pm 7.1$ & $19.8 \pm 7.8$ & $0.510^{\mathrm{t}}$ \\
\hline Data & &
\end{tabular}

Data are presented as mean \pm standard deviation

tStudent's $t$ test, "Wilcoxon tes

TDO: total displacement oscillation, ACPap: amplitude of displacement of the center of pressure in the anterior-posterior plane, ACPml: amplitude of

displacement of the center of pressure in the mid-lateral plane, AREA: displacement area, AAS: anterior-posterior average speed, MAS: mid-lateral average speed,

TAS: total average speed

stabilometric variables demonstrated increased stability and static balance in this group after 12 weeks of exercise. Some studies have in dicated that the muscles respond to application of the PNF hold-relax stretching technique, suggesting that in addition to promoting gains in flexibility and increases in the range of motion, PNF also increases the muscle strength of the knee flexor and extensor muscles [26]. Furthermore, during the execution of the PNF movements, the muscles are briefly stretched before contraction, stimulating neuromuscular endings that produce greater strength. When exercises are performed using a diagonal pattern, which is parallel to the muscle topography, more physiological and functional movements are reproduced, such as gait [27]. The lower-limb PNF exercises were performed using the bilaterally symmetrical antagonist pattern, 
Table 5 Functional tests of elderly women before and after (within-group comparison) Pilates and PNF training or usual activity (control)

\begin{tabular}{llll}
\hline & Pre & Post & $p^{*}$ \\
\hline Control & & & \\
BERG & $50.5 \pm 3.4$ & $51.0 \pm 3.5$ & 0.462 \\
TAF (cm) & $19.7 \pm 7.6$ & $20.0 \pm 6.5$ & 0.878 \\
TUG (sec) & $13.8 \pm 3.3$ & $13.9 \pm 4.3$ & 0.915 \\
PNF & & & \\
BERG & $51.7 \pm 4.8$ & $55.8 \pm 0.4$ & 0.001 \\
TAF (cm) & $21.7 \pm 7.3$ & $30.9 \pm 5.7$ & $<0.001$ \\
TUG (sec) & $10.7 \pm 1.9$ & $8.1 \pm 1.9$ & $<0.001$ \\
Pilates & & & \\
BERG & $54.0 \pm 1.9$ & $56.0 \pm 0.1$ & $<0.001$ \\
TAF (cm) & $24.6 \pm 7.0$ & $33.4 \pm 5.9$ & $<0.001$ \\
TUG (sec) & $11.3 \pm 2.5$ & $7.7 \pm 1.5$ & $<0.001$ \\
\hline Data & & &
\end{tabular}

Data are presented as mean \pm standard deviation

*Significant difference by Student's $t$ test

BERG: Berg Balance Scale score, TAF: functional reach test, TUG: timed up and go test

which not only strengthens the muscles involved in gait, but also allows for irradiation of the most powerful member to facilitate the movements of weaker muscles [9].

Among the reductions observed in the stabilometric parameters in the PNFG, the COP variables in the midlateral plane (ACPml and MAS) achieved statistical significance in the PNFG, while a significant reduction was not observed in any COP parameter in the anterior-posterior plane. This may have been a consequence of the use of a diagonal pattern, which allows the exercises to always be performed in combination with hip abduction and adduction and ankle inversion and eversion, stimulating the afferent somatosensory and lateral and middle muscular groups of the lower limbs and conferring greater stability to the mid-lateral plane.

Although no studies in the literature have utilized stabilometric variables to evaluate balance in elderly women after PNF exercises, Pereira et al. [7] evaluated the risk of falling among elderly people after PNF exercises were performed three times a week for 10 weeks. They verified significant improvements in the Berg Balance Scale scores and greater strength in the extensor compartment of the knee. Cilento et al. [8] also conducted a prospective study to comparatively evaluate the effectiveness of three training protocols in elderly women randomly selected and divided into four groups: a traditional muscle strengthening group, functional circuit training group, PNF exercise group, and control group. The elderly women participated in 30-min sessions twice a week for 10 weeks. At the end of the 10 weeks, they were evaluated using the TUG test and functional reach test. Statistically significant improvement was observed in all groups compared with the control group. In the present study, women in the PNFG exhibited significant improvements in the Berg Balance Scale scores, functional reach test results, and TUG test results.

Some studies have suggested that a better balance between agonist and antagonist muscle activation is achieved after PNF exercises, reducing coactivation [9, 27]. Research shows that increased coactivation around the knee and ankle is associated with greater strength and lower torque in elderly people $[7,27,28]$. Coactivation, defined as the simultaneous contraction of agonist and antagonist muscles around a joint, is responsible for reductions in walking speed and increases in the risk of falls in the elderly $[28,29]$. Therefore, we can explain the improvement in static balance through the reductions in the stabilometric parameters and the improvement in dynamic balance through the improvements in the Berg Balance Scale scores, TUG test performance, and functional reach test performance in the elderly women after the PNF exercise protocol in the present study.

Another common exercise currently recommended and used in this study is the Pilates method, which emphasizes principles such as strengthening of the abdominal and paraspinal muscles in parallel with good posture, breathing, and body alignment [30]. The Pilates method effectively improves postural stability in elderly individuals because it emphasizes trunk control [14]. A strong and stable trunk can contribute to more efficient use of the upper and lower limbs and thus better balance and functional performance in older adults [4].

Although women in the PG did not show significant improvements in the COP parameters, they exhibited improvements in the performance of functional tests for dynamic balance. The exercise protocol for this method challenges balance. According to Sherrington et al. [5, 31], exercises performed for fall prevention should provide a moderate or high challenge to balance. Moreover, this technique helps to strengthen the abdominal muscles [32], which are important for a good posture. The strengthened abdominal muscles reduce anterior pelvic inclination, common in this age group, thus improving gait [33].

In a randomized study, Bird et al. [15] evaluated the static and dynamic conditions of elderly residents in a community sample through evaluation of the ACPml and performance of the TUG test, comparing the Pilates method with a control method. No significant improvement was observed in any measured COP variables between the two groups. However, within-group comparison revealed an increase in the TUG test performance $(p<$ 0.001 ) in the Pilates group. Likewise, in the present study, there was a significant improvement in dynamic balance.

Kaesler et al. [12] examined the efficacy of a Pilates program specifically designed to improve balance. The sample comprised eight elderly adults (four female and 
four male). The sessions were performed twice a week for 8 weeks. As in the present study, no significant improvement was found in $\mathrm{COP}$ on a firm surface. However, the researchers verified better performance on the TUG test after the proposed treatment. Newell et al. [13] executed Pilates training with nine elderly adults (eight female and one male) once a week for 8 weeks. There was no significant difference in the COP body sway pre- and post-treatment $(p>0.05)$, but there was an increase in the walking speed, cycle, and stride length. Mokhtari et al. [14] found a significant improvement in the TUG test and functional reach test performance among elderly adults who participated in Pilates three times a week for 12 weeks when compared with a control group.

Postural balance results from complex interactions among the sensory, nervous, and musculoskeletal systems and requires constant adaptations of muscular activity, the articular position, and other sensorial information to maintain the center of body mass on the support base [34]. Pilates exercises are based on movement control, which can lead to changes in the nervous system through alterations in synaptic connections and cortical remapping [35].

The results of the present study demonstrated improvements compared with the CG, particularly in dynamic balance in the PG and static and dynamic balance in the PNFG. This may have occurred because the PNF exercises were performed with manual resistance provided by a therapist, eliciting maximum motor recruitment through the stretch reflex, manual contact, verbal stimulation, force irradiation, and visual-auditory biofeedback, all of which improve sensory compensation in the elderly as suggested by previous clinical studies $[9,10]$. Pilates is an exercise method that requires contraction and coordination of multiple muscle groups to achieve better motor recruitment, as well as synchronization of body movements and breathing [11]. Thus, the elderly may need a longer duration and greater number of sessions to learn and maximally benefit from the Pilates protocol.

Furthermore, as this is a per protocol analysis reflecting the benefits of these two exercise methods in a predetermined population, the effect of the interventions can not be applied to real life scenarios.

\section{Conclusions}

Women in the Proprioceptive Neuromuscular FacilitationGroup demonstrated improvement in postural static stability as shown by evaluation of various stabilometric parameters. We also found a significant increase in dynamic balance as verified by the functional test performance in the Pilates and Proprioceptive Neuromuscular Facilitation groups. After only 4 weeks of these exercises, an improvement in balance could be observed, although without differences between the Pilates and Proprioceptive Neuromuscular Facilitation groups. Therefore, we recommend that further studies include larger samples of elderly women and greater numbers of sessions. This will help to elucidate the optimal alternatives that can be applied to increase balance, allowing PNF and Pilates exercises to be used not only for rehabilitation, but also as a preventive method.

\section{Abbreviations}

PNFG: Proprioceptive neuromuscular facilitation group; PG: Pilates group; CG: Control group; TDO: Total displacement oscillation; ACPap: Amplitude of displacement of the center of pressure in the anterior-posterior plane; ACPml: Amplitude of displacement of the center of pressure in the mid-lateral plane; AREA: Displacement area; AAS: Anterior-posterior average speed; MAS: Mid-lateral average speed; TAS: Total average speed; TUG test: Timed up and go test.

\section{Competing interests}

The authors declare that they have no competing interests.

\section{Authors' contributions}

LSAM was the primary researcher and designed the study together with FTC, acquired the data with LSAF, OPN, and RAZ, performed the statistical analysis, and drafted the manuscript. OPN and RAZ reviewed the manuscript. All authors read and approved the final version of the manuscript.

\section{Acknowledgements}

This research was conducted with financial support provided by FAPESP-Fundação de Amparo à Pesquisa do Estado de São Paulo, Process: 2012/09400-9.

\section{Author details}

'Universidade Camilo Castelo Branco -Unicastelo, São Paulo, SP, Brazil. ${ }^{2}$ Associação Cidade da Ciência Tecnologia e Educação -CITÉ, São José dos Campos, SP, Brazil. ' Universidade Estadual do Piauí- UESPI, Teresina, PI, Brazil. ${ }^{4}$ Centro Universitário Uninovafapi, Teresina, PI, Brazil. ${ }^{5}$ Rua Industrial Francisco Castro, 1622, Bairro Horto, Teresina, PI, Brazil.

Received: 23 December 2014 Accepted: 21 May 2015

Published online: 02 June 2015

\section{References}

1. Duarte M, Freitas SMSF. Revisão sobre posturografia baseada em plataforma de força para avaliação do equilíbrio. Revis Bras Fisiot. 2010;14(3):183-92.

2. ParreiraRB ACF, Gil AW, Teixeira DC, Bilodeau M, Silva RA. Effect of trunk extensor fatigue on the postural balance of elderly and young adults during unipodal task. Eur J Appl Physiol. 2013;113(8):1989-96.

3. Mann L, Kleinpaul JF, Mota CB, Santos SG. Equilíbrio corporal e exercícios físicos: uma revisão sistemática. Revista Motriz. 2009;15:713-22.

4. Granacher U, Gollhofer A, Hortobagyi T, Kressig RWK, Muehlbauer T. The importance of trunk muscle strength for balance, functional performance, and fall prevention in seniors: a systematic review. Sports Med. 2013:43:627-41.

5. Sherrington C, Tiedemann A, Fairhall N, Close JCT, Lord SR. Exercise to prevent falls in older adults: an updated meta-analysis and best practice recommendations. N S W Public Health Bull. 2011;22:78-83.

6. Romero-Franco N, Martinez-Lopez E, Lomas-Vega R, Hita-Contreras F, Osuna-Pérez MC, Martínez-Amat A. Short-term effects of proprioceptive training with unstable platform on athletes' stabilometry. J Strength Cond Res. 2013;27:2189-97.

7. Pereira MPP, Gonçalves M. Proprioceptive neuromuscular facilitation improves balance and knee extensors strength of older fallers. ISRN Rehabil. 2012;402612:7

8. Cilento MBR, Nóbrega ACL, Araújo AQC. Avaliação da eficácia de protocolos de treinamento da atividade sentado-para-de-pé em mulheres idosas. Fisioter Bras. 2005:6:412-8.

9. Adler SS, Beckers D, Buck M. PNF in practice: an illustrated guide. Verlag Berlin Heidelberg: Springer; 2014.

10. Witt D, Talbott N, Kotowski S. Electromyographic activity of scapular muscles during diagonal patterns using elastic resistance and free weights. Int J Sports PhysTher. 2011;6:322. 
11. Latey P. Updating the principles of the Pilates method. J Bodyw Mov Ther. 2002;6:94-101.

12. Kaesler DS, Mellifont RB, Swete KP, Taaffe DR. A novel balance exercise program for postural stability in older adults: a pilot study. J Bodyw Mov Ther. 2007;11:37-43.

13. Newell D, Shead V, Sloane L. Changes in gait and balance parameters in elderly subjects attending an 8-week supervised Pilatesprogramme. J Bodyw Mov Ther. 2012;16:549-54.

14. Mokhtari M, Nezakatalhossaini M, Esfarjani F. The effect of 12-week pilates exercises on depression and balance associated with falling in the elderly. Procedia Soc Behav Sci. 2013;70:1714-23.

15. Bird ML, Hill BKD, Fell JWFA. Randomized controlled study investigating static and dynamic balance in older adults after training with pilates. Arch Phys Med Rehabil. 2012;93(1):43-9.

16. Rees SS, Murphy AJ, Watsford ML. Effects of vibration exercise on muscle performance and mobility in an older population. J Aging Phys Act. 2007;15:367-81.

17. Teixeira CS, Lemos LFC, Lopes LFD, Rossi AG, Mota CB. Equilíbrio corporal e exercícios físicos: uma investigação com mulheres idosas praticantes de diferentes modalidades. Acta Fisiátrica. 2008;15:156-9.

18. Podsiadlo D, Richardson S. The timed "Up \& Go": a test of basic functional mobility for frail elderly persons. J Am Geriatr Soc. 1999;39:142-8.

19. Duncan PW, Weiner DK, Chandler J, Studenski S. Functional reach: a new clinical measure of balance. J Gerontol. 1990;45:M192-7.

20. Miyamoto ST, Lombardi Jr I, Berg KO, Ramos LR, Natour J. Brazilian version of the Berg balance scale. Braz J Med Biol Res. 2004;37:1411-4.

21. Thais BA, Nélida AS, Juliana NC, Marcio MP, Marisete PS. Efeito da equoterapia no equilíbrio postural de idosos. Rev Bras Fisioter. 2011;15:414-9.

22. Fujimoto $C$, Murofushi $T$, Chihara $Y$, Ushio M, Sugasawa $K$, Yamaguchi $T$, et al. Assessment of diagnostic accuracy of foam posturography for peripheral vestibular disorders: analysis of parameters related to visual and somatosensory dependence. Clin Neurophysiol. 2009;120:1408-14.

23. Ruhe A, Fejer R, Walker B. The test-retest reliability of centre of pressure measures in bipedal static task conditions - a systematic review of the literature. Gait Posture. 2010;32:436-45.

24. Paes FJV, Silva RA, Freira ALG, Politti F, Chiavegato L, Geraldes AR, et al. Immediate effect of bilateral talocrural joint manipulation on postural balance in healthy subjects. J Ergon. 2013;3:122.

25. Silva RA, Bilodeau M, Parreira RB, Teixeira DC, Amorim CF. Age related differences in time-limit performance and force platform-based balance measures during one-leg stance. J Electromyogr Kinesiol. 2013;23:634-9.

26. Rees SS, Murphy AJ, Watsford ML, Mclachlan KA, Coutts AJ. Effects of proprioceptive neuromuscular facilitation stretching on stiffness and force-producing characteristics of the ankle in active women. J Strength Cond Res. 2007;21:572-7.

27. Kofotolis N, Kellis E. Effects of two 4-week proprioceptive neuromuscular facilitation programs on muscle endurance, flexibility, and functional performance in women with chronic low back pain. Phys Ther. 2006;86:1001-12.

28. Lee HJ, Chou LS. Detection of gait instability using the center of mass and center of pressure inclination angles. Arch Phys Med Rehabil. 2006:87:569-75.

29. Pereira MP, Gonçalves M. Muscular coactivation (CA) around the knee reduces power production in elderly women. Arch Gerontol Geriatr. 2011;52:317-21.

30. Kawanishi CY, Oliveira MR, Coelho VS, Parreira RB, Oliveira RF, Santos RB, et al. Efeitos dos exercícios pilates na função do tronco e na dor de pacientes com lombalgia. Revista Terapia Manual. 2011;9:410-4.

31. Sherrington C, Whitney JC, Lord SR, Herbert RD, Cumming RG, Close JCT. Effective exercise for the prevention of falls: a systematic review and meta-analysis. JAGS. 2008;56:2234-46.

32. Kloubec JA. Pilates for improvement of muscle endurance, flexibility, balance, and posture. J Strength Cond Res. 2010;24:661-7.

33. Freitas EV. Tratado de geriatria e gerontologia. 3rd ed. Rio de Janeiro: Guanabara Koogan; 2011.

34. Vernazza-Martin S, Tricon V, Martin N, Mesure S, Azulay J, Le Pellec-Muller A. Effect of aging on the coordination between equilibrium and movement: what changes? Exp Brain Res. 2008;187(2):255-65.

35. Bolognini N, Pascual-Leone A, Fregni F. Using non-invasive brain stimulation to augment motor training-induced plasticity. J Neuroeng Rehabil. 2009;6:1-13

\section{Submit your next manuscript to BioMed Central and take full advantage of:}

- Convenient online submission

- Thorough peer review

- No space constraints or color figure charges

- Immediate publication on acceptance

- Inclusion in PubMed, CAS, Scopus and Google Scholar

- Research which is freely available for redistribution

Submit your manuscript at www.biomedcentral.com/submit 